\title{
Evaluation of Comprehensive 2D Gas Chromatography-Time-of-Flight Mass Spectrometry for 209 Chlorinated Biphenyl Congeners in Two Chromatographic Runs
}

\author{
L. I. Osemwengie and G. W. Sovocool \\ National Exposure Research Laboratory Environmental Sciences Division, U.S. Environmental Protection Agency,
} P.O. Box 93478, Las Vegas, NV 89193-3478, USA

Correspondence should be addressed to L. I. Osemwengie, osemwengie.lantis@epa.gov

Received 29 July 2010; Accepted 28 February 2011

Academic Editor: Encarna Moyano

Copyright (C) 2011 L. I. Osemwengie and G. W. Sovocool. This is an open access article distributed under the Creative Commons Attribution License, which permits unrestricted use, distribution, and reproduction in any medium, provided the original work is properly cited.

This research evaluates a recently developed comprehensive two-dimensional gas chromatography 2D GC coupled with a time-of-flight (TOF) mass spectrometer for the potential separation of 209 PCB congeners, using a sequence of 1D and 2D chromatographic modes. In two consecutive chromatographic runs, using a $40 \mathrm{~m}$, Rtx-PCB column, and a $1 \mathrm{~m}$ DB-17 column, connected in series, 196 PCB congeners are distinguished, including 43 of the 46 pentachlorobiphenyl isomers. Some of the chlorinated biphenyls that could not be resolved chromatographically are resolved with the use of the "ortho effect," which distinguishes PCB isomers having 2,2' -; and 2,2' 6- chlorine substitution from those isomers without these substitutions. The result of this work falls short of our goal of separating all 209 PCB congeners but still provides investigators with a new tool for a better front-end separation of PCB-specific congeners, and potentially, for use in acquisition of more accurate data.

\section{Introduction}

Chlorinated biphenyls (CBs), or polychlorinated biphenyls (PCBs), are comprised of 209 distinct chlorine-substituted biphenyl structures (congeners). Ten isomeric groups of congeners exist with varying degrees of chlorination (see Table 1). Approximately 140 to 150 of the 209 PCB congeners listed in Table 2 were found in the complex mixtures (Aroclors) that were used commercially in a variety of applications, including heat transfer and hydraulic fluids, dielectric fluids for capacitors, and as additives in pesticides, sealants, and plastics [1-3]. The dispersion of PCB congeners in the form of the Aroclors by uncontrolled release into the environment, their long-term stability, and possible toxicity, together caused concern for their biological and environmental impact. The World Health Organization (WHO) designated twelve PCBs as "dioxin-like, coplanar PCB congeners" that exhibited high toxicity [4]. Originally, the noncoplanar PCBs were considered the most toxic because they were present in much greater abundances. Later, the "coplanar" PCBs were found to have dioxin-like toxicity and received the most attention. It appears that the toxicity of the noncoplanar PCBs has been re-emphasized because most noncoplanar congeners with ortho chlorine substitution were also found to be toxic in mammalian brains

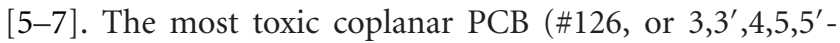
pentachlorobiphenyl) was known to coelute with $2,3,7,8$ dibenzo-p-dioxin [8], and with PCB (\#159 or $2,3,3^{\prime}, 4,5,5^{\prime}-$ hexachlorobiphenyl) in environmental samples using the DB-XLB phase. High resolution mass spectrometry (HRMS) was usually required to resolve PCB 126 and 2,3,7,8-dibenzop-dioxin, when both were present in environmental samples in varying concentrations [9]. Tandem mass spectrometry (MS/MS) can also be used for distinguishing dioxins from PCBs. Dioxins can lose $\cdot \mathrm{COCl}$ whereas PCBs can only show chlorine losses. To permit congener specific environmental 
TAвLE 1: Polychlorinated biphenyl Congener classes with identification ions.

\begin{tabular}{|c|c|c|c|c|c|}
\hline PCB Congener Classes & $\begin{array}{c}\text { Numbers of PCB isomers } \\
\text { per congener class }\end{array}$ & Molecular Formula & Identification ions $\mathrm{M}^{+} \bullet$ & $(\mathrm{M}-\mathrm{Cl})^{+}$ & $(\mathrm{M}-2 \mathrm{Cl})^{+\bullet}$ \\
\hline Monochlorobiphenyl & 3 & $\mathrm{C}_{12} \mathrm{H}_{9} \mathrm{Cl}$ & 188 & 153 & - \\
\hline Dichlorobiphenyl & 12 & $\mathrm{C}_{12} \mathrm{H}_{8} \mathrm{Cl}_{2}$ & 222 & 187 & 152 \\
\hline Trichlorobiphenyl & 24 & $\mathrm{C}_{12} \mathrm{H}_{7} \mathrm{Cl}_{3}$ & 256 & 221 & 186 \\
\hline Tetrachlorobiphenyl & 42 & $\mathrm{C}_{12} \mathrm{H}_{6} \mathrm{Cl}_{4}$ & $290(292)^{*}$ & 255 & 220 \\
\hline Pentachlorobiphenyl & 46 & $\mathrm{C}_{12} \mathrm{H}_{5} \mathrm{Cl}_{5}$ & $324(326)$ & $289(291)^{*}$ & 254 \\
\hline Hexachlorobiphenyl & 42 & $\mathrm{C}_{12} \mathrm{H}_{4} \mathrm{Cl}_{6}$ & $358(360)$ & $323(325)$ & $288(290)^{*}$ \\
\hline Heptachlorobiphenyl & 24 & $\mathrm{C}_{12} \mathrm{H}_{3} \mathrm{Cl}_{7}$ & $392(394)$ & $357(359)$ & $322(324)$ \\
\hline Octachlorobiphenyl & 12 & $\mathrm{C}_{12} \mathrm{H}_{2} \mathrm{Cl}_{8}$ & $426(430)$ & $391(393)$ & $356(358)$ \\
\hline Nonachlorobiphenyl & 3 & $\mathrm{C}_{12} \mathrm{HCl}_{9}$ & $460(464)$ & $425(429)$ & $390(392)$ \\
\hline Decachlorobiphenyl & 1 & $\mathrm{C}_{12} \mathrm{Cl}_{10}$ & $494(498)$ & $459(463)$ & $424(428)$ \\
\hline
\end{tabular}

* Most abundant ion of the isotope cluster is in parentheses, if not first member.

analysis of the PCBs, scientists invested considerable research effort towards optimizing the gas chromatographic conditions and techniques required for separations on various capillary columns [10-12]. Frame has published work on 20 different capillary columns with various stationary phases [12]. Their work provided a database of retention time information for various columns that analysts could use as a reference for PCB column choice and methodology. More recently, Reiner et al. and Cochran demonstrated the use of fast gas chromatography coupled with time-of-flight mass spectrometry for the analysis of PCB congeners $[13,14]$. Their work demonstrated the use of narrow bore capillary columns and the fast data acquisition rates ( 50 spectra/s) of a time-of-flight mass spectrometer (TOFMS) for the identification of PCB congeners in minimal analysis time. Taking advantage of the full mass range data acquisition of the TOFMS allowed for peak identification and spectral deconvolution of overlapping peaks present in the mixtures.

The Contract Laboratory Program (CLP) that provided analytical support for Superfund, measured PCB congeners in commercial, or environmentally altered mixtures, using high-resolution gas chromatography (HRGC), combined with detectors such as the electron capture detector (ECD), and high-resolution mass spectrometer (HRMS) [4, 1517]. The GC/ECD was unable to distinguish target compounds from many interfering, coextracted impurities [18]. Therefore, CLP results from GC/ECD could potentially be associated with overestimation or misidentification of $\mathrm{PCB}$ congeners. U.S. EPA Method 1668a required HRGC/HRMS for the determination of 137 chlorinated biphenyl congeners, including the twelve WHO toxic chlorinated biphenyl congeners in water, soil, sediment, biosolids, tissue, and other sample matrices [4]. The remaining 72 chlorinated biphenyl congeners were either not present in the Aroclors or determined as mixtures of isomers or congeners from GC coelution. Other drawbacks of HRGC/HRMS were the high costs of acquisition, maintenance, sample analysis, and required operation by highly trained personnel.

Due to the observation of close-eluting and some poorly resolved chlorinated biphenyls in the use of GC/ECD with the 30-m Rtx-5 column, previous investigators of congenerspecific PCBs in fish reported combined values for PCB congener pairs with IUPAC numbers 61/74, 77/110, 82/151, $156 / 202$, and $194 / 205$ in lake trout, whitefish, and walleye collected from the Great Lakes, and selected inland lakes in the United States [19]. There were many other examples of similar coelutions in the literature, depending on the column phase and experimental conditions [2, 3]. The resulting combined congener values increased the difficulty of defining exposure and of making ecosystem health assessments.

In recent years, comprehensive two-dimensional gas chromatography (GC $\times$ GC) or $2 \mathrm{D}-\mathrm{GC}$ was successfully coupled to TOFMS for a variety of complex sample analyses, such as chlorinated hydrocarbons, and mixtures of environmental analytes [20-25]. A review of comprehensive twodimensional gas chromatography by Beens and Brinkman in 2005 showed that the separation of chlorinated biphenyls in fish extract, using GC $\times$ GC without the necessary summing software for several modulations per analyte, was much improved compared to 1D-GC separation [26]. A goal of this study was to attempt to separate and unambiguously distinguish all of the 209 chlorinated biphenyl congeners in two different chromatographic runs using 1D-GC, with the thermal modulator deactivated in the first run and activated in the second run for 2D-GC.

$\mathrm{GC} \times \mathrm{GC}$ is a relatively new technique [27], yet to be adapted by the CLP program. Since its inception, many researchers have published reviews and experimental results for a variety of complex samples using GC $\times \mathrm{GC}-\mathrm{ECD}$ or $\mathrm{GC} \times \mathrm{GC}-\mu \mathrm{ECD}$, and more recently GC $\times \mathrm{GC}$-TOFMS $[8,20$, $21,24,28,29]$, due to the complex nature of a single run in the $2 \mathrm{D}$ chromatographic separation method, their studies 
TABLE 2: Polychlorinated biphenyl congener number, IUPAC names, CAS numbers, and retention times in increasing order, using sequential $40 \mathrm{~m}$ Rtx-PCB and $1 \mathrm{~m}$ DB-17 GC columns (1D Mode).

\begin{tabular}{|c|c|c|c|}
\hline Congener no. & IUPAC name & CASRN & Retention time (s) \\
\hline 1 & 2-chlorobiphenyl & $2051-60-7$ & 1395.45 \\
\hline 2 & 3-chlorobiphenyl & 2051-61-8 & 1705.70 \\
\hline 3 & 4-chlorobiphenyl & $2051-62-9$ & 1777.55 \\
\hline 4 & $2,2^{\prime}$-dichlorobiphenyl & $13029-08-8$ & 1838.05 \\
\hline 10 & 2,6-dichlorobiphenyl & $33146-45-1$ & 1840.75 \\
\hline 9 & 2,5-dichlorobiphenyl & $34883-39-1$ & 2090.25 \\
\hline 7 & 2,4-dichlorobiphenyl- & $33284-50-3$ & 2103.35 \\
\hline 6 & 2,3'-dichlorobiphenyl & $25569-80-6$ & 2171.75 \\
\hline 5 & 2,3-dichlorobiphenyl & $16605-91-7$ & 2232.15 \\
\hline 8 & 2,4'-dichlorobiphenyl & $34883-43-7$ & 2261.70 \\
\hline 19 & 2,2',6-trichlorobiphenyl & $38444-73-4$ & 2381.25 \\
\hline 14 & 3,5-dichlorobiphenyl- & $34883-41-5$ & 2413.30 \\
\hline 30 & 2,4,6-trichlorobiphenyl & $35693-92-6$ & 2476.60 \\
\hline 18 & 2,2',5-trichlorobiphenyl & $37680-65-2$ & 2613.05 \\
\hline 11 & $3,3^{\prime}$-dichlorobiphenyl & $2050-67-1$ & 2628.35 \\
\hline 17 & $2,2^{\prime}, 4$-trichlorobiphenyl & $37680-66-3$ & 2640.00 \\
\hline 12 & 3,4-dichlorobiphenyl & $2974-92-7$ & 2683.85 \\
\hline 27 & 2,3',6-trichlorobiphenyl & $38444-76-7$ & 2699.35 \\
\hline 24 & 2,3,6-trichlorobiphenyl & $55702-45-9$ & 2735.45 \\
\hline 13 & $3,4^{\prime}$-dichlorobiphenyl & $2974-90-5$ & 2737.55 \\
\hline 16 & 2,2',3-trichlorobiphenyl & $38444-78-9$ & 2789.25 \\
\hline 32 & 2,4',6-trichlorobiphenyl & $38444-77-4$ & 2826.00 \\
\hline 15 & $4,4^{\prime}$-dichlorobiphenyl & $2050-68-2$ & 2854.10 \\
\hline 34 & $2,3^{\prime}, 5^{\prime}$-trichlorobiphenyl & $37680-68-5$ & 2903.00 \\
\hline 54 & $2,2^{\prime}, 6,6^{\prime}$-tetrachlorobiphenyl & $15968-05-5$ & 2910.50 \\
\hline 23 & 2,3,5-trichlorobiphenyl & $55720-44-0$ & 2926.35 \\
\hline 29 & 2,4,5-trichlorobiphenyl & $15862-07-4$ & 2961.45 \\
\hline 50 & 2,2',4,6-tetrachlorobiphenyl & $62796-65-0$ & 3041.90 \\
\hline 26 & $2,3^{\prime}, 5$-trichlorobiphenyl & $38444-81-4$ & 3058.20 \\
\hline 25 & $2,3^{\prime}, 4$-trichlorobiphenyl & $55712-37-3$ & 3086.65 \\
\hline 53 & $2,2^{\prime}, 5,6^{\prime}$-tetrachlorobiphenyl & $41464-41-9$ & 3164.85 \\
\hline 31 & 2,4',5-trichlorobiphenyl & $16606-02-3$ & 3181.00 \\
\hline 28 & $2,4,4^{\prime}$-trichlorobiphenyl & $7012-37-5$ & 3210.95 \\
\hline 33 & $2,3^{\prime}, 4^{\prime}$-trichlorobiphenyl & $38444-86-9$ & 3217.00 \\
\hline 21 & 2,3,4-trichlorobiphenyl & $55702-46-0$ & 3218.20 \\
\hline 51 & $2,2^{\prime}, 4,6^{\prime}$-tetrachlorobiphenyl & $68194-04-7$ & 3226.80 \\
\hline 20 & 2,3,3' -trichlorobiphenyl & $38444-84-7$ & 3227.75 \\
\hline 45 & 2,2',3,6-tetrachlorobiphenyl & $70362-45-7$ & 3312.35 \\
\hline 22 & 2,3,4' -trichlorobiphenyl & $38444-85-8$ & 3353.85 \\
\hline 46 & $2,2^{\prime}, 3,6^{\prime}$-tetrachlorobiphenyl & $41464-47-5$ & 3372.70 \\
\hline 73 & $2,3^{\prime}, 5^{\prime}, 6$-tetrachlorobiphenyl & 74338-23-1 & 3395.50 \\
\hline 69 & 2,3',4,6-tetrachlorobiphenyl & $60233-24-1$ & 3440.00 \\
\hline 43 & 2,2',3,5-tetrachlorobiphenyl & $70362-46-8$ & 3450.95 \\
\hline 36 & 3,3',5-trichlorobiphenyl & $38444-87-0$ & 3463.00 \\
\hline 52 & $2,2^{\prime}, 5,5^{\prime}$-tetrachlorobiphenyl & $35693-99-3$ & 3485.35 \\
\hline 48 & 2,2',4,5-tetrachlorobiphenyl & $70362-47-9$ & 3498.60 \\
\hline
\end{tabular}


Table 2: Continued.

\begin{tabular}{|c|c|c|c|}
\hline Congener no. & IUPAC name & CASRN & Retention time $(\mathrm{s})$ \\
\hline 49 & $2,2^{\prime}, 4,5^{\prime}$-tetrachlorobiphenyl & $41464-40-8$ & 3531.35 \\
\hline 104 & $2,2^{\prime}, 4,6,6^{\prime}$-pentachlorobiphenyl & $56558-16-8$ & 3550.00 \\
\hline 65 & 2,3,5,6-tetrachlorobiphenyl & $33284-54-7$ & 3570.50 \\
\hline 62 & 2,3,4,6-tetrachlorobiphenyl & $54230-22-7$ & 3574.50 \\
\hline 47 & $2,2^{\prime}, 4,4^{\prime}$-tetrachlorobiphenyl & $2437-79-8$ & 3574.95 \\
\hline 75 & 2,4,4',6-tetrachlorobiphenyl & $32598-12-2$ & 3592.50 \\
\hline 39 & 3,4',5-trichlorobiphenyl & $38444-88-1$ & 3599.50 \\
\hline 38 & 3,4,5-trichlorobiphenyl & $53555-66-1$ & 3602.50 \\
\hline 44 & $2,2^{\prime}, 3,5^{\prime}$-tetrachlorobiphenyl & $41464-39-5$ & 3678.75 \\
\hline 59 & 2,3,3',6-tetrachlorobiphenyl & $74472-33-6$ & 3714.60 \\
\hline 42 & $2,2^{\prime}, 3,4^{\prime}$-tetrachlorobiphenyl & $36559-22-5$ & 3726.90 \\
\hline 71 & $2,3^{\prime}, 4^{\prime}, 6$-tetrachlorobiphenyl & 41464-46-4 & 3765.00 \\
\hline 35 & 3,3',4-trichlorobiphenyl & $37680-69-6$ & 3782.80 \\
\hline 41 & 2,2',3,4-tetrachlorobiphenyl & $52663-59-9$ & 3791.60 \\
\hline 96 & 2,2',3,6,6' -pentachlorobiphenyl & $73575-54-9$ & 3814.40 \\
\hline 72 & $2,3^{\prime}, 5,5^{\prime}$-tetrachlorobiphenyl & $41464-42-0$ & 3850.00 \\
\hline 103 & 2,2',4,5',6-pentachlorobiphenyl & $60145-21-3$ & 3864.50 \\
\hline 64 & 2,3,4',6-tetrachlorobiphenyl & $52663-58-8$ & 3872.50 \\
\hline 40 & $2,2^{\prime}, 3,3^{\prime}$-tetrachlorobiphenyl & $38444-93-8$ & 3880.25 \\
\hline 68 & $2,3^{\prime}, 4,5^{\prime}$-tetrachlorobiphenyl & $73575-52-7$ & 3893.50 \\
\hline 37 & $3,4,4^{\prime}$-Trichlorobiphenyl & $38444-90-5$ & 3923.00 \\
\hline 100 & 2,2',4,4',6-pentachlorobiphenyl & $39485-83-1$ & 3939.00 \\
\hline 94 & 2,2',3,5,6' -pentachlorobiphenyl & $73575-55-0$ & 3952.25 \\
\hline 57 & 2,3,3',5-tetrachlorobiphenyl & $70424-67-8$ & 3974.00 \\
\hline 67 & 2,3',4,5-tetrachlorobiphenyl & $73557-53-8$ & 4025.50 \\
\hline 58 & $2,3,3^{\prime}, 5^{\prime}$-tetrachlorobiphenyl & 41464-49-7 & 4026.00 \\
\hline 102 & $2,2^{\prime}, 4,5,6^{\prime}$-pentachlorobiphenyl & 68194-06-9 & 4030.00 \\
\hline 61 & 2,3,4,5-tetrachlorobiphenyl & $33284-53-6$ & 4061.50 \\
\hline 98 & $2,2^{\prime}, 3,4^{\prime}, 6^{\prime}$-pentachlorobiphenyl & $60233-25-2$ & 4077.00 \\
\hline 93 & $2,2^{\prime}, 3,5,6$-pentachlorobiphenyl & $73575-56-1$ & 4092.95 \\
\hline 76 & $2,3^{\prime}, 4^{\prime}, 5^{\prime}$-tetrachlorobiphenyl & $70362-48-0$ & 4100.00 \\
\hline 63 & 2,3,4',5-tetrachlorobiphenyl & $74472-34-7$ & 4124.50 \\
\hline 88 & $2,2^{\prime}, 3,4,6$-pentachlorobiphenyl & $55215-17-3$ & 4127.20 \\
\hline 95 & $2,2^{\prime}, 3,5^{\prime}, 6$-pentachlorobiphenyl & $38379-99-6$ & 4136.40 \\
\hline 121 & $2,3^{\prime}, 4,5^{\prime}, 6$-pentachlorobiphenyl & $56558-18-0$ & 4155.00 \\
\hline 74 & 2,4,4',5-tetrachlorobiphenyl & $32690-93-0$ & 4168.50 \\
\hline 155 & $2,2^{\prime}, 4,4^{\prime}, 6,6^{\prime}$-hexachlorobiphenyl & $33979-03-2$ & 4194.50 \\
\hline 70 & $2,3^{\prime}, 4^{\prime}, 5$-tetrachlorobiphenyl & $32598-11-1$ & 4199.50 \\
\hline 91 & $2,2^{\prime}, 3,4^{\prime}, 6$-pentachlorobiphenyl & $68194-05-8$ & 4216.70 \\
\hline 66 & $2,3^{\prime}, 4,4^{\prime}$-tetrachlorobiphenyl & $32598-10-0$ & 4240.50 \\
\hline 55 & 2,3,3',4,-tetrachlorobiphenyl & $74338-24-2$ & 4304.00 \\
\hline 80 & $3,3^{\prime}, 5,5^{\prime}$-tetrachlorobiphenyl & $33284-52-5$ & 4312.50 \\
\hline 92 & $2,2^{\prime}, 3,5,5^{\prime}$-pentachlorobiphenyl & $52663-61-3$ & 4342.75 \\
\hline 89 & 2,2',3,4,6' -pentachlorobiphenyl & $73575-57-2$ & 4352.60 \\
\hline 84 & $2,2^{\prime}, 3,3^{\prime}, 6$-pentachlorobiphenyl & $52663-60-2$ & 4360.40 \\
\hline 56 & $2,3,3^{\prime}, 4^{\prime}$-tetrachlorobiphenyl & $41464-43-1$ & 4378.00 \\
\hline 90 & $2,2^{\prime}, 3,4^{\prime}, 5$-pentachlorobiphenyl & 68194-07-0 & 4404.40 \\
\hline 101 & $2,2^{\prime}, 4,5,5^{\prime}$-pentachlorobiphenyl & $37680-73-2$ & 4407.40 \\
\hline 113 & 2,3,3', ,5',6-pentachlorobiphenyl & $68194-10-5$ & 4421.00 \\
\hline 60 & 2,3,4,4' -tetrachlorobiphenyl & $33025-41-1$ & 4446.50 \\
\hline
\end{tabular}


Table 2: Continued.

\begin{tabular}{|c|c|c|c|}
\hline Congener no. & IUPAC name & CASRN & Retention time $(\mathrm{s})$ \\
\hline 150 & $2,2^{\prime}, 3,4^{\prime}, 6,6^{\prime}$-hexachlorobiphenyl & 68194-08-1 & 4457.00 \\
\hline 99 & $2,2^{\prime}, 4,4^{\prime}, 5$-pentachlorobiphenyl & 38380-01-7 & 4460.00 \\
\hline 152 & $2,2^{\prime}, 3,5,6,6^{\prime}$-hexachlorobiphenyl & 68194-09-2 & 4507.00 \\
\hline 119 & $2,3^{\prime}, 4,4^{\prime}, 6$-pentachlorobiphenyl & $56558-17-9$ & 4543.00 \\
\hline 83 & $2,2^{\prime}, 3,3^{\prime}, 5$-pentachlorobiphenyl & $60145-20-2$ & 4546.00 \\
\hline 125 & $2,3^{\prime}, 4^{\prime}, 5^{\prime}, 6$-pentachlorobiphenyl & $74472-39-2$ & 4558.00 \\
\hline 86 & $2,2^{\prime}, 3,4,5$-pentachlorobiphenyl & $55312-69-1$ & 4560.15 \\
\hline 112 & 2,3,3',5,6-pentachlorobiphenyl & $74472-36-9$ & 4562.50 \\
\hline 145 & $2,2^{\prime}, 3,4,6,6^{\prime}$-hexachlorobiphenyl & $74472-40-5$ & 4578.00 \\
\hline 108 & 2,3,3',4,6-pentachlorobiphenyl & $74472-35-8$ & 4584.50 \\
\hline 97 & $2,2^{\prime}, 3^{\prime}, 4,5$-pentachlorobiphenyl & 41464-51-1 & 4603.00 \\
\hline 148 & $2,2^{\prime}, 3,4^{\prime}, 5,6^{\prime}$-hexachlorobiphenyl & $74472-41-6$ & 4644.00 \\
\hline 116 & 2,3,4,5,6-pentachlorobiphenyl & 18259-05-7 & 4653.00 \\
\hline 79 & $3,3^{\prime}, 4,5^{\prime}$-tetrachlorobiphenyl & $41464-48-6$ & 4654.50 \\
\hline 87 & $2,2^{\prime}, 3,4,5^{\prime}$-pentachlorobiphenyl & $38380-02-8$ & 4712.25 \\
\hline 78 & 3,3',4,5-tetrachlorobiphenyl & $70362-49-1$ & 4720.50 \\
\hline 136 & $2,2^{\prime}, 3,3^{\prime}, 6,6^{\prime}$-hexachlorobiphenyl & $38411-22-2$ & 4722.90 \\
\hline 154 & $2,2^{\prime}, 4,4^{\prime}, 5,6^{\prime}$-hexachlorobiphenyl & $60145-22-4$ & 4731.50 \\
\hline 117 & 2,3,4',5,6-pentachlorobiphenyl & 68194-11-6 & 4744.50 \\
\hline 115 & 2,3,4,4',6-pentachlorobiphenyl & $74472-38-1$ & 4752.00 \\
\hline 111 & $2,3,3^{\prime}, 5,5^{\prime}$-pentachlorobiphenyl & $39635-32-0$ & 4757.00 \\
\hline 85 & $2,2^{\prime}, 3,4,4^{\prime}$-pentachlorobiphenyl & $65510-45-4$ & 4767.15 \\
\hline 110 & 2,3,3',4',6-pentachlorobiphenyl & 38380-03-9 & 4818.50 \\
\hline 120 & $2,3^{\prime}, 4,5,5^{\prime}$-pentachlorobiphenyl & $68194-12-7$ & 4822.50 \\
\hline 81 & $3,4,4^{\prime}, 5$-tetrachlorobiphenyl & $70362-50-4$ & 4873.50 \\
\hline 151 & $2,2^{\prime}, 3,5,5^{\prime}, 6$-hexachlorobiphenyl & $52663-63-5$ & 4893.50 \\
\hline 135 & $2,2^{\prime}, 3,3^{\prime}, 5,6^{\prime}$-hexachlorobiphenyl & $52744-13-5$ & 4913.95 \\
\hline 82 & $2,2^{\prime}, 3,3^{\prime}, 4$-pentachlorobiphenyl & $52663-62-4$ & 4915.25 \\
\hline 144 & $2,2^{\prime}, 3,4,5^{\prime}, 6$-hexachlorobiphenyl & $68194-14-9$ & 4946.00 \\
\hline 147 & $2,2^{\prime}, 3,4^{\prime}, 5,6$-hexachlorobiphenyl & $68194-13-8$ & 4990.60 \\
\hline 77 & $3,3^{\prime}, 4,4^{\prime}$-tetrachlorobiphenyl & $32598-13-3$ & 4998.50 \\
\hline 149 & $2,2^{\prime}, 3,4^{\prime}, 5^{\prime}, 6$-hexachlorobiphenyl & $38380-04-0$ & 4999.50 \\
\hline 143 & $2,2^{\prime}, 3,4,5,6^{\prime}$-hexachlorobiphenyl & 68194-15-0 & 5018.00 \\
\hline 139 & $2,2^{\prime}, 3,4,4^{\prime}, 6$-hexachlorobiphenyl & $56030-56-9$ & 5030.85 \\
\hline 140 & $2,2^{\prime}, 3,4,4^{\prime}, 6^{\prime}$-hexachlorobiphenyl & 59291-64-4 & 5057.35 \\
\hline 124 & $2,3^{\prime}, 4^{\prime}, 5,5^{\prime}$-pentachlorobiphenyl & 70424-70-3 & 5068.50 \\
\hline 107 & $2,3,3^{\prime}, 4,5^{\prime}$-pentachlorobiphenyl & $70362-41-3$ & 5106.00 \\
\hline 123 & $2^{\prime}, 3,4,4^{\prime}, 5$-pentachlorobiphenyl & $65510-44-3$ & 5118.00 \\
\hline 188 & $2,2^{\prime}, 3,4^{\prime}, 5,6,6^{\prime}$-heptachlorobiphenyl & $74487-85-7$ & 5122.00 \\
\hline 134 & $2,2^{\prime}, 3,3^{\prime}, 5,6$-hexachlorobiphenyl & $52704-70-8$ & 5124.65 \\
\hline 109 & $2,3,3^{\prime}, 4^{\prime}, 5$-pentachlorobiphenyl & $70424-68-9$ & 5128.00 \\
\hline 106 & 2,3,3',4,5-pentachlorobiphenyl & 70424-69-0 & 5134.50 \\
\hline 142 & 2,2',3,4,5,6-hexachlorobiphenyl & 41411-61-4 & 5135.00 \\
\hline 131 & 2,2',3,3',4,6-hexachlorobiphenyl & 61798-70-7 & 5167.60 \\
\hline 133 & $2,2^{\prime}, 3,3^{\prime}, 5,5^{\prime}$-hexachlorobiphenyl & $35694-04-3$ & 5176.15 \\
\hline 118 & $2,3^{\prime}, 4,4^{\prime}, 5$-pentachlorobiphenyl & $31508-00-6$ & 5185.50 \\
\hline 184 & $2,2^{\prime}, 3,4,4^{\prime}, 6,6^{\prime}$-heptachlorobiphenyl & $74472-48-3$ & 5202.45 \\
\hline 165 & $2,3,3^{\prime}, 5,5^{\prime}, 6$-hexachlorobiphenyl & $74472-46-1$ & 5233.50 \\
\hline 122 & $2,3,3^{\prime}, 4^{\prime}, 5^{\prime}$-pentachlorobiphenyl & 76842-07-4 & 5246.50 \\
\hline
\end{tabular}


TABle 2: Continued.

\begin{tabular}{|c|c|c|c|}
\hline Congener no. & IUPAC name & CASRN & Retention time (s) \\
\hline 146 & $2,2^{\prime}, 3,4^{\prime}, 5,5^{\prime}$-hexachlorobiphenyl & 51908-16-8 & 5249.00 \\
\hline 161 & $2,3,3^{\prime}, 4,5^{\prime}, 6$-hexachlorobiphenyl & $74474-43-8$ & 5275.50 \\
\hline 114 & 2,3,4,4',5-pentachlorobiphenyl & $74472-37-0$ & 5289.50 \\
\hline 168 & $2,3^{\prime}, 4,4^{\prime}, 5^{\prime}, 6$-hexachlorobiphenyl & 59291-65-5 & 5310.50 \\
\hline 153 & $2,2^{\prime}, 4,4^{\prime}, 5,5^{\prime}$-hexachlorobiphenyl & $35065-27-1$ & 5324.00 \\
\hline 132 & $2,2^{\prime}, 3,3^{\prime}, 4,6^{\prime}$-hexachlorobiphenyl & $38380-05-1$ & 5333.85 \\
\hline 179 & $2,2^{\prime}, 3,3^{\prime}, 5,6,6^{\prime}$-heptachlorobiphenyl & $52663-64-6$ & 5383.30 \\
\hline 141 & $2,2^{\prime}, 3,4,5,5^{\prime}$-hexachlorobiphenyl & $52712-04-6$ & 5448.05 \\
\hline 176 & $2,2^{\prime}, 3,3^{\prime}, 4,6,6^{\prime}$-heptachlorobiphenyl & $52663-65-7$ & 5463.10 \\
\hline 105 & 2,3,3',4,4' -pentachlorobiphenyl & $32598-14-4$ & 5469.00 \\
\hline 186 & $2,2^{\prime}, 3,4,5,6,6^{\prime}$-heptachlorobiphenyl & $74472-49-4$ & 5500.00 \\
\hline 137 & $2,2^{\prime}, 3,4,4^{\prime}, 5$-hexachlorobiphenyl & $35694-06-5$ & 5511.10 \\
\hline 127 & $3,3^{\prime}, 4,5,5^{\prime}$-pentachlorobiphenyl & $39635-33-1$ & 5552.00 \\
\hline 130 & $2,2^{\prime}, 3,3^{\prime}, 4,5^{\prime}$-hexachlorobiphenyl & $52663-66-8$ & 5566.30 \\
\hline 164 & $2,3,3^{\prime}, 4^{\prime}, 5^{\prime}, 6$-hexachlorobiphenyl & $74472-45-0$ & 5574.00 \\
\hline 138 & $2,2^{\prime}, 3,4,4^{\prime}, 5^{\prime}$-hexachlorobiphenyl & $35065-28-2$ & 5627.80 \\
\hline 178 & $2,2^{\prime}, 3,3^{\prime}, 5,5^{\prime}, 6$-heptachlorobiphenyl & $52663-67-9$ & 5632.50 \\
\hline 160 & 2,3,3',4,5,6-hexachlorobiphenyl & $41411-62-5$ & 5637.00 \\
\hline 163 & 2,3,3', $4^{\prime}, 5,6$-hexachlorobiphenyl & $74472-44-9$ & 5648.00 \\
\hline 129 & $2,2^{\prime}, 3,3^{\prime}, 4,5$-hexachlorobiphenyl & $55215-18-4$ & 5648.45 \\
\hline 158 & 2,3,3',4,4',6-hexachlorobiphenyl & $74472-42-7$ & 5677.00 \\
\hline 182 & $2,2^{\prime}, 3,4,4^{\prime}, 5,6^{\prime}$-heptachlorobiphenyl & $60145-23-5$ & 5690.05 \\
\hline 175 & $2,2^{\prime}, 3,3^{\prime}, 4,5^{\prime}, 6$-heptachlorobiphenyl & 40186-70-7 & 5695.20 \\
\hline 187 & $2,2^{\prime}, 3,4^{\prime}, 5,5^{\prime}, 6$-heptachlorobiphenyl & $52663-68-0$ & 5725.00 \\
\hline 183 & $2,2^{\prime}, 3,4,4^{\prime}, 5^{\prime}, 6$-heptachlorobiphenyl & $52663-69-1$ & 5787.85 \\
\hline 166 & 2,3,4,4',5,6-hexachlorobiphenyl & $41411-63-6$ & 5818.00 \\
\hline 159 & $2,3,3^{\prime}, 4,5,5^{\prime}$-hexachlorobiphenyl & $39635-35-3$ & 5893.00 \\
\hline 126 & $3,3^{\prime}, 4,4^{\prime}, 5$-pentachlorobiphenyl & $57465-28-8$ & 5906.50 \\
\hline 185 & $2,2^{\prime}, 3,4,5,5^{\prime}, 6$-heptachlorobiphenyl & $52712-05-7$ & 5915.00 \\
\hline 128 & $2,2^{\prime}, 3,3^{\prime}, 4,4^{\prime}$-hexachlorobiphenyl & $38380-07-3$ & 5937.80 \\
\hline 162 & $2,3,3^{\prime}, 4^{\prime}, 5,5^{\prime}$-hexachlorobiphenyl & $39635-34-2$ & 5951.00 \\
\hline 174 & $2,2^{\prime}, 3,3^{\prime}, 4,5,6^{\prime}$-heptachlorobiphenyl & $38411-25-5$ & 5953.45 \\
\hline 202 & $2,2^{\prime}, 3,3^{\prime}, 5,5^{\prime}, 6,6^{\prime}$-octachlorobiphenyl & $2136-99-4$ & 5998.55 \\
\hline 181 & $2,2^{\prime}, 3,4,4^{\prime}, 5,6$-heptachlorobiphenyl & $74472-47-2$ & 6009.70 \\
\hline 167 & $2,3^{\prime}, 4,4^{\prime}, 5,5^{\prime}$-hexachlorobiphenyl & $52663-72-6$ & 6023.00 \\
\hline 177 & $2,2^{\prime}, 3,3^{\prime}, 4,5^{\prime}, 6^{\prime}$-heptachlorobiphenyl & $52663-70-4$ & 6069.60 \\
\hline 201 & $2,2^{\prime}, 3,3^{\prime}, 4,5^{\prime}, 6,6^{\prime}$-octachlorobiphenyl & $52663-73-7$ & 6087.50 \\
\hline 204 & $2,2^{\prime}, 3,4,4^{\prime}, 5,6,6^{\prime}$-octachlorobiphenyl & $74472-52-9$ & 6087.60 \\
\hline 171 & $2,2^{\prime}, 3,3^{\prime}, 4,4^{\prime}, 6$-heptachlorobiphenyl & $52663-71-5$ & 6117.90 \\
\hline 173 & $2,2^{\prime}, 3,3^{\prime}, 4,5,6$-heptachlorobiphenyl & 68194-16-1 & 6139.75 \\
\hline 197 & $2,2^{\prime}, 3,3^{\prime}, 4,4^{\prime}, 6,6^{\prime}$-octachlorobiphenyl & $33091-17-7$ & 6174.95 \\
\hline 172 & $2,2^{\prime}, 3,3^{\prime}, 4,5,5^{\prime}$-heptachlorobiphenyl & $52663-74-8$ & 6252.10 \\
\hline 156 & 2,3,3',4,4',5-hexachlorobiphenyl & $38380-08-4$ & 6267.00 \\
\hline 192 & $2,3,3^{\prime}, 4,5,5^{\prime}, 6$-heptachlorobiphenyl & $74472-51-8$ & 6281.00 \\
\hline 157 & $2,3,3^{\prime}, 4,4^{\prime}, 5^{\prime}$-hexachlorobiphenyl & $69782-90-7$ & 6304.50 \\
\hline 180 & $2,2^{\prime}, 3,4,4^{\prime}, 5,5^{\prime}$-heptachlorobiphenyl & $35065-29-3$ & 6330.60 \\
\hline 200 & $2,2^{\prime}, 3,3^{\prime}, 4,5,6,6^{\prime}$-octachlorobiphenyl & $52663-75-9$ & 6341.45 \\
\hline 193 & 2,3,3', $4^{\prime}, 5,5^{\prime}, 6$-heptachlorobiphenyl & $69782-91-8$ & 6348.50 \\
\hline
\end{tabular}


TABle 2: Continued.

\begin{tabular}{|c|c|c|c|}
\hline Congener no. & IUPAC name & CASRN & Retention time $(\mathrm{s})$ \\
\hline 191 & $2,3,3^{\prime}, 4,4^{\prime}, 5^{\prime}, 6$-heptachlorobiphenyl & $74472-50-7$ & 6399.00 \\
\hline 198 & $2,2^{\prime}, 3,3^{\prime}, 4,5,5^{\prime}, 6$-octachlorobiphenyl & $68194-17-2$ & 6610.75 \\
\hline 199 & $2,2^{\prime}, 3,3^{\prime}, 4,5,5^{\prime}, 6^{\prime}$-octachlorobiphenyl & 40186-71-8 & 6624.80 \\
\hline 170 & $2,2^{\prime}, 3,3^{\prime}, 4,4^{\prime}, 5$-heptachlorobiphenyl & $35065-30-6$ & 6626.15 \\
\hline 190 & $2,3,3^{\prime}, 4,4^{\prime}, 5,6$-heptachlorobiphenyl & $41411-64-7$ & 6670.50 \\
\hline 196 & $2,2^{\prime}, 3,3^{\prime}, 4,4^{\prime}, 5,6^{\prime}$-octachlorobiphenyl & $42740-50-1$ & 6682.60 \\
\hline 203 & $2,2^{\prime}, 3,4,4^{\prime}, 5,5^{\prime}, 6$-octachlorobiphenyl & $52663-76-0$ & 6691.25 \\
\hline 169 & $3,3^{\prime}, 4,4^{\prime}, 5,5^{\prime}$-hexachlorobiphenyl & $32774-16-6$ & 6724.00 \\
\hline 208 & $2,2^{\prime}, 3,3^{\prime}, 4,5,5^{\prime}, 6,6^{\prime}$-nonachlorobiphenyl & $52663-77-1$ & 6835.70 \\
\hline 207 & $2,2^{\prime}, 3,3^{\prime}, 4,4^{\prime}, 5,6,6^{\prime}$-nonachlorobiphenyl & $52663-79-3$ & 6893.05 \\
\hline 195 & $2,2^{\prime}, 3,3^{\prime}, 4,4^{\prime}, 5,6$-octachlorobiphenyl & $52663-78-2$ & 6915.85 \\
\hline 189 & $2,3,3^{\prime}, 4,4^{\prime}, 5,5^{\prime}$-heptachlorobiphenyl & $39635-31-9$ & 6917.20 \\
\hline 194 & $2,2^{\prime}, 3,3^{\prime}, 4,4^{\prime}, 5,5^{\prime}$-octachlorobiphenyl & $35694-08-7$ & 7071.45 \\
\hline 205 & $2,3,3^{\prime}, 4,4^{\prime}, 5,5^{\prime}, 6$-octachlorobiphenyl & $74472-53-0$ & 7121.15 \\
\hline 206 & $2,2^{\prime}, 3,3^{\prime}, 4,4^{\prime}, 5,5^{\prime}, 6$-nonachlorobiphenyl & $40186-72-9$ & 7288.20 \\
\hline 209 & decachlorobiphenyl & 2051-24-3 & 7471.40 \\
\hline
\end{tabular}

have not sufficiently resulted in the transfer of the 2D-GC technology to the commercial industry.

\section{Experimental}

The GC $\times$ GC-TOFMS instrument used for this experiment was the liquid nitrogen quad-jet modulator, with two nozzles for heating and two for cooling, Pegasus 4D (Leco Corp., St Joseph, MI, USA). The modulator is installed between the 1D and 2D columns in an Agilent 6890 GC oven (Palo Alto, CA, USA). The system is equipped with a secondary oven, and a 6890 series auto-injector. Liquid nitrogen and breathable air are the sources of the cold and hot jets, respectively. The detector is a time-of-flight mass analyzer with a setup, which can be described as postacceleration, where all ions leave the drift region with approximately $900 \mathrm{eV}$ kinetic energy, and then accelerate to $2000 \mathrm{eV}$ or higher before striking the microchannel plate surface. The first column used in this study was a nonpolar phase RtxPCB (proprietary Crossbond phase) GC column (Restek, Bellefonte, PA, USA), of 40 meters length, $0.18 \mathrm{~mm} \mathrm{ID}$, and $0.18 \mu \mathrm{m}$ film thickness, while the second column was a polar phase DB-17 (cross-linked/surface bonded 50\% phenyl, 50\% methylpolysiloxane) GC column, (Agilent Technologies, Palo Alto, CA, USA), of 1 meter length, $0.10 \mathrm{~mm} \mathrm{ID,} \mathrm{and}$ $0.10 \mu \mathrm{m}$ film thickness.

\section{Materials and Method}

Two hundred and nine certified standard solutions of individual chlorinated biphenyls (International Union of Pure and Applied Chemistry (IUPAC) \# 1 through 209) in isooctane were used for this study (see Table 2) and were purchased from AccuStandard, Inc., (New Haven, CT, USA).
Each isooctane solution contained $100 \mathrm{ng} / \mu \mathrm{L}$ of an individual chlorinated biphenyl congener and was diluted 5-fold with 99.9\% $n$-hexane (B\&J GC2 grade, Burdick and Jackson, Muskegon, MI, USA) to produce $20 \mathrm{ng} / \mu \mathrm{L}$. To compensate for the observed decreasing signals of the more highly chlorinated congeners, the concentrations of the injected congener solutions were adjusted to between 0.20 and $4.25 \mathrm{ng} / \mu \mathrm{L}$. The concentration adjustments yielded approximately equal signal intensities upon analysis by GC $\times$ GC-TOFMS.

In $\mathrm{GC} \times \mathrm{GC}$-TOFMS, the injected sample was transferred from the first column into the second column, aided by means of a thermal-based modulator. The GC $\times$ GC column configurations as used here consisted of a long first dimension (1D) column, at $40 \mathrm{~m}$ in length and containing a proprietary phase that separated compounds based upon their volatility, that is, with retention times increasing with compound boiling points [30]. A nonpolar phase GC colu$\mathrm{mn}$ was connected with a press-fit connector (Varian universal quick seal, Varian-Chrompack, Palo Alto, CA, USA) serially to a second, but shorter (about $1 \mathrm{~m}$ ), polar phase GC column. After several temperature program cycles, a DB-17 GC column was determined to be optimal for the PCB separations due to its thermal stability $\left(280^{\circ} \mathrm{C}\right)$ at the GC $\times$ GC-TOFMS interface.

The modulator period was $4 \mathrm{~s}$ with the hot-pulse duration set at $1200 \mathrm{~ms}$ and the cool time between modulation stages set at $800 \mathrm{~ms}$. When a $2 \mathrm{D}$ run was begun, the modulator operated throughout the entire analysis and performed uninterrupted trapping and releasing of the effluent from the primary column. The GC $\times$ GC-TOFMS system may also be operated in the $1 \mathrm{D}$ mode by deactivating the thermal modulator. The functions of the thermal modulator have been described elsewhere $[8,27,31-33]$. Data was continuously acquired by the high speed detector (TOFMS) 
and deconvoluted by commercially available software (ChromaTOF), that came with the instrument, which generated contour plots, as well as three-dimensional chromatograms, based upon the user defined modulation period. The TOFMS detector was utilized because the fast chromatographic technique that generated multiple narrow peaks from the short secondary column required a fast scanning detector, capable of producing sufficient data points to accurately define a chromatographic peak, and to deconvolute nearly overlapping peaks. The first GC run used both columns in the GC configured in a one-dimensional mode of separation (with the modulator turned off) to define where more separation using the 2D mode of separation (with the modulator turned on) was needed. The second GC run was configured in a two-dimensional mode of separation, to distinguish some of the most difficult to separate coeluting PCB congeners. Successful separation and measurement of specific PCB congeners held potential for significant improvements in estimating environmental exposure to $\mathrm{PCB}$ congeners and toxicological evaluations of PCB congeners in biological tissues.

\section{GC $\times$ GC-TOFMS Analysis of Chlorinated Biphenyl Congeners}

The GC $\times$ GC-TOFMS chromatographic conditions were optimized as follows: initial primary oven temperature containing the first column was set at $70^{\circ} \mathrm{C}$ held for $0.5 \mathrm{~min}$, ramped at $10^{\circ} \mathrm{C} / \mathrm{min}$ to $150^{\circ} \mathrm{C}$, then at $1^{\circ} \mathrm{C} / \mathrm{min}$ to $250^{\circ} \mathrm{C}$, followed by a $4^{\circ} \mathrm{C} / \mathrm{min}$ ramp to $275^{\circ} \mathrm{C}$ and held for $15 \mathrm{~min}$. The second-dimension column in the secondary oven ran with a $15^{\circ} \mathrm{C}$ offset to the primary oven temperature. Using ultra pure helium as carrier gas, the target flow rate was set at a corrected constant flow of $1.20 \mathrm{~mL} / \mathrm{min}$. The inlet temperature was set at $280^{\circ} \mathrm{C}$ for splitless injection of $1 \mu \mathrm{L}$. The conditions above allowed for the early elution of volatile compounds, which would eliminate them as potential background interferences. Instrument control (data acquisition) and data processing (postacquisition) were conducted with commercially available software-the integrated Leco ChromaTOF. Mass spectrometric conditions were set as follows: the filament was turned on at 1100s into the run, until the end. The mass range was 45 to $550 \mathrm{amu}$, mass acquisition rate at 3 spectra/s (1D mode), and 100 spectra/s (2D mode), detector voltage at $1.65 \mathrm{KV}$, electron energy at $70 \mathrm{~V}$, transfer line temperature at $280^{\circ} \mathrm{C}$, ion source temperature set at $200^{\circ} \mathrm{C}$.

\section{Relative Retention Time and Mass Spectral Library for all 209 PCB Congeners}

In this research, one PCB congener was taken from each homologue group to create a solution. Exactly 46 solutions were created to bracket all 46 pentachlorobiphenyl isomers. The first solution contained 10 congeners, the second and third contained 9 each, the fourth through twelfth contained 7 each, and so forth. For the purpose of general matching between congener classes, a mass spectral library of 209 PCB individual congeners was created using 46 different solutions, containing unique groups of the $\mathrm{PCB}$ congeners. The design was such that no two isomeric PCBs were included in any of the 46 solutions. All 46 solutions were individually analyzed (46 chromatographic runs) using the $1 \mathrm{D}$ mode (thermal modulation deactivated) because most of the congeners (188) were separated by the 1D mode. The instrument conditions for the 1D mode of separation were the same as previously described above, except that the thermal modulator was not activated. The PCB standard used in this research was constructed as follows: (Congener number range, chlorine number, concentration in $\mathrm{ng} / \mu \mathrm{L}$; PCBs 1-3 (1Cl), 0.20; PCBs 4-15 (2Cl), 0.40; PCBs 16-39 (3Cl), 0.85; PCB 40 (4Cl), 1.25; PCBs 41-81 (4Cl), 1.50; PCB 82 (5Cl), 1.50; PCBs 83-127 (5Cl), 2.00; PCBs 128-169 (6Cl), 3.00; PCB 170 (7Cl), 3.40; PCBs 171-193 (7Cl), 4.00; PCB 194 (8Cl), 3.25; PCBs 195-196 (8Cl), 4.05; PCBs 197-205 (8Cl), 4.25; PCB 206 (9Cl), 3.25; PCBs 207-208 (9Cl), 4.00; and PCB $209(10 \mathrm{Cl}), 3.00)$. The first solution contained the following ten congeners of IUPAC numbers: $1,4,16,40,82,128,170,194,206$, and 209 , using individual concentration as shown above. The second solution contained nine congeners of IUPAC numbers: 2, 5, 17, 41, $83,129,171,195$, and 207. One congener was taken from each of the ten chlorination levels or homologue groups each time until 46 different solutions of congeners were achieved. Each congener was chromatographically separated in each of the mixtures, and this enabled the identification of every member of the group by molecular weight and chlorine isotope cluster. As each run was completed, the file was manually transferred to a dedicated workstation computer, for postacquisition data processing, while the instrumentdedicated computer system continued to acquire raw data. In this way, using the same chromatographic conditions, several runs were made each day, and retention times and mass spectra were archived for all of the congeners. Some of the data acquired with 50 scans/s in the $1 \mathrm{D}$ chromatographic mode were resampled using the default data resampling function of the instrument to smooth the data.

During a preliminary chromatographic run of the first solution containing mono through decachlorobiphenyl congeners, in the 1D mode, it was noticed that there was a sharp decrease in peak signal or intensity from the early eluting $\mathrm{PCB}$ congeners to the later eluting ones, with decachlorobiphenyl's ion chromatogram being extremely small. Due to the fact that this TOFMS is equipped with a postacceleration system, we therefore attributed this phenomenon to the decreasing molar concentration for a given weight of sample, as the molecular weight increased, and the larger number of fragments of the more highly chlorinated PCB congeners, resulting in more ions with low intensity. It thus became necessary to adjust the concentration of each component within the ten chlorination levels or first solution of ten PCB congeners. This achieved approximately equivalent signals for each component by increasing the concentrations of the later, higher molecular weight eluters. Also, to raise the sensitivity level for the purpose of detecting higher masses, auto-tuning was performed using $\mathrm{m} / \mathrm{z} 414$ from perfluorotributylamine (PFTBA) instead of the default $\mathrm{m} / z 69$. 


\section{Analysis of the 209 Polychlorinated Biphenyl Mixture in the 2D Mode}

A combined solution of 9 ampoules containing a mixture of all 209 PCB congeners at adjusted concentration of $6 \mathrm{ng} / \mu \mathrm{L}$ (AccuStandard, New Haven, CT, USA), was analyzed using two different separation modes-1D and 2D chromatography.

Experimental conditions were the same as previously described above. The versatility of the $2 \mathrm{D}$ chromatographic mode in this research was of major importance because it was used to separate additional pentachlorobiphenyls to the 188 congeners that were distinguished using the 1D chromatographic mode. It may be optimized and used for the separation of those congeners previously or partially separated in the $1 \mathrm{D}$ chromatographic mode for isomers with great concentration differentials.

\section{Results and Discussion}

The chemical identity of all PCB congeners rested primarily on the accuracy of the individual labeled ampoules supplied by AccuStandard Inc. (New Haven, CT, USA), for the creation of our mass spectral library. However, all individual PCB congeners were also verified by mass spectrometry as providing ions consistent with Table 1 and as having the correct chlorine isotope clusters. Only 140 of the PCB congeners were available in the National Institute of Standards and Technology (NIST '05) mass spectral library [34]. The information recorded from the acquired full-scan data was used to create a new mass spectral library for all 209 PCB congeners. The intensities of key ions from this newly created mass spectral library were compared with those from the mass spectra available in the NIST '05 library. This 209 congener library provided an opportunity to include and test another parameter relating chemical structures and the mass spectra, with the potential to help distinguish isomers. This was the mass spectrometric "ortho effect" observed for chlorinated biphenyls having 2,2'-; and $2,2^{\prime}, 6$-chlorine substitution, and to a much lesser extent, $2,2^{\prime} 6,6^{\prime}$-substitution [35-38]. This effect showed itself as an increase, for the above ortho substituted isomers and congeners, of the chlorine cluster resulting from the loss of the first chlorine $(\mathrm{M}-\mathrm{Cl})^{+}$relative to the chlorine cluster containing the molecular ion, $\mathrm{M}^{+\bullet}$. PCBs having 2,6-diortho substitution on the same ring, or no ortho substitution at all, showed quite different spectra, with no or very small losses of the first chlorine. All standards were therefore also checked for consistency of the structure with the presence or absence of the expected "ortho-effect," and with the spectra present in the NIST '05 Library. As a result of this crosschecking, it was also observed that congener IUPAC number 99 (2,2' 4,4'5-pentachlorobiphenyl) yielded an "ortho effect" consistent with $2,2^{\prime}$-chlorine substitution for our standard $(20 \%)$, but inconsistent with the value in the NIST library (1\%), that would be characteristic of no ortho, or 2,6di-ortho substitution. Similarly, congeners 19 and 41 were found to be in error. We believe these library spectra were mislabeled, as revealed by our complete PCB library.

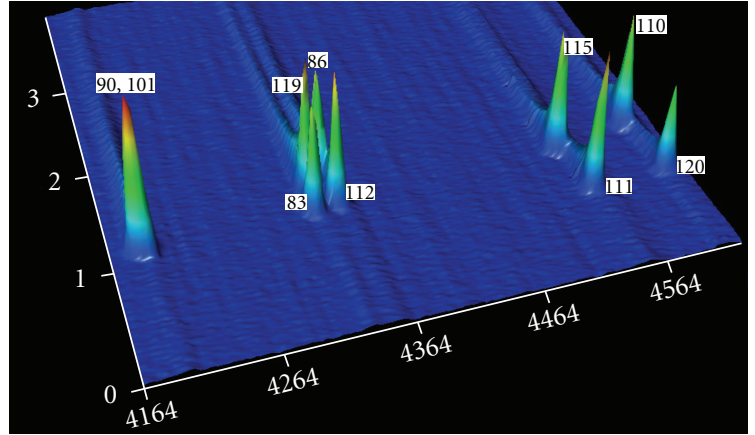

FIGURE 1: Surface view showing the use of $2 \mathrm{D}$ in the separation of four pairs of polychlorinated biphenyl isomers with IUPAC numbers beginning from the right of $(110+120),(115+111),(86$ $+112)$, and $(119+83)$. The PCB isomers (left pair) numbers $(90+$ 101) were inseparable under the chromatographic conditions used. Both axes are in seconds.

The GC retention properties of the standards were also checked against several literature references [12,39-41]. For this comparison, a few earlier structural assignments were corrected to the modern IUPAC numbers [42, 43]. The structures and retention properties for all 209 congeners were listed in Table 2.

Of the 209 possible PCB congeners, the 1D gas chromatographic mode of separation was able to distinguish 188 PCB congeners from their distinct GC peaks, and from the use of selected ion current profiles, or mass chromatograms, for resolving coeluting congeners with their different molecular weights. Most of the coeluting isomers were from the 46 isomeric pentachlorobiphenyls, and these were arranged in order of their elution in Table 3 with coeluting groups designated with the letters c through $\mathrm{g}$ and which consisted of PCBs of IUPAC numbers: $(90+101)$, $(83+119),(86+112),(115+111)$, and $(110+120)$. All of these five isomer pairs in parentheses eluted within a few seconds of each other, which appeared to be about the minimum possible separation by $\mathrm{GC} \times \mathrm{GC}$-TOFMS in the 1D chromatographic mode. Therefore, the five pairs could not be separated in the $1 \mathrm{D}$ chromatographic mode, but were identified by their retention times from their individual 1D chromatographic runs of the authentic standards in Table 2 . The members of the pairs $(83+119)$ and $(86+112)$ were also distinguished from each other by use of the mass spectrometric "ortho effect;" the contrasting values of these were also given in the right-hand column of Table 3. Eight of the ten pentachlorobiphenyl isomers that could not be separated by $1 \mathrm{D}$ were separated by $2 \mathrm{D}$ (Figure 1 ). The $2 \mathrm{D}$ chromatographic run also showed separation of penta isomer 125 from isomers 86 and 112 .

The remaining two penta PCBs with IUPAC numbers 90 and 101 could not be separated with the 2D mode. Because both of these penta isomers had 2,2'-ortho substitution, their respective "ortho effects" were also too similar to allow them to be distinguished. However, the 2D separation mode distinguished the tetra isomers 55 and 80 . Difficulty was encountered in the separation of a pair of dichlorobiphenyls. 
Table 3: GC Elution (1D Mode) and MS “Ortho Effect” of the Forty-six Penta CBs.

\begin{tabular}{|c|c|c|c|c|}
\hline Elution Order & IUPAC \# & Structure Ring 1 : Ring 2 & RT $\sec ^{\mathrm{a}}$ & "Ortho Effect" \%" \\
\hline 1 & 104 & $246: 26$ & 3550 & 10 \\
\hline 2 & 96 & $236: 26$ & 3814 & $13 \mathrm{~h}$ \\
\hline 3 & 103 & $246: 25$ & 3864 & $38 \mathrm{~h}$ \\
\hline 4 & 100 & $246: 24$ & 3939 & 31 \\
\hline 5 & 94 & $235: 26$ & 3952 & 50 \\
\hline 6 & 102 & $245: 26$ & 4030 & 36 \\
\hline 7 & 98 & $246: 23$ & 4077 & 51 \\
\hline 8 & 93 & $2356: 2$ & 4093 & 59 \\
\hline 9 & 88 & $2346: 2$ & 4127 & 48 \\
\hline 10 & 95 & $236: 25$ & 4136 & $46 \mathrm{~h}$ \\
\hline 11 & 121 & $246: 35$ & 4155 & $6 \mathrm{~h}$ \\
\hline 12 & 91 & $236: 24$ & 4217 & $42 \mathrm{~h}$ \\
\hline 13 & 92 & $235: 25$ & 4343 & 49 \\
\hline 14 & 89 & $234: 26$ & 4353 & 51 \\
\hline 15 & 84 & $236: 23$ & 4360 & 63 \\
\hline 16 & 90 & $235: 24$ & 4404 c & 42 \\
\hline 17 & 101 & $245: 25$ & 4407 c & 29 \\
\hline 18 & 113 & $236: 35$ & 4421 & $7 \mathrm{~h}$ \\
\hline 19 & 99 & $245: 24$ & 4460 & $20 \mathrm{~h}$ \\
\hline 20 & 119 & $246: 34$ & $4543 \mathrm{~d}$ & $3 \mathbf{h}$ \\
\hline 21 & 83 & $235: 23$ & $4546 \mathrm{~d}$ & $69 \mathrm{~h}$ \\
\hline 22 & 125 & $345: 26$ & 4558 e & $7 \mathbf{h}$ \\
\hline 23 & 86 & $2345: 2$ & 4560 e & $44 \mathrm{~h}$ \\
\hline 24 & 112 & $2356: 3$ & 4562 e & $6 \mathrm{~h}$ \\
\hline 25 & 108 & $2346: 3$ & 4584 & 4 \\
\hline 26 & 97 & $245: 23$ & 4603 & $38 \mathrm{~h}$ \\
\hline 27 & 116 & 23456 & 4653 & $6 \mathrm{~h}$ \\
\hline 28 & 87 & $234: 25$ & 4712 & $41 \mathrm{~h}$ \\
\hline 29 & 117 & $2356: 4$ & 4744 & $4 \mathrm{~h}$ \\
\hline 30 & 115 & $2346: 4$ & $4752 \mathrm{f}$ & 3 \\
\hline 31 & 111 & $235: 35$ & $4757 \mathrm{f}$ & 3 \\
\hline 32 & 85 & $234: 24$ & 4767 & $33 \mathrm{~h}$ \\
\hline 33 & 110 & $236: 34$ & $4818 \mathrm{~g}$ & $3 \mathrm{~h}$ \\
\hline 34 & 120 & $245: 35$ & $4822 \mathrm{~g}$ & 3 \\
\hline 35 & 82 & $234: 23$ & 4915 & $54 \mathrm{~h}$ \\
\hline 36 & 124 & $345: 25$ & 5068 & $5 \mathrm{~h}$ \\
\hline 37 & 107 & $234: 35$ & 5106 & 3 \\
\hline 38 & 123 & $345: 24$ & 5118 & 3 \\
\hline 39 & 109 & $235: 34$ & 5128 & 3 \\
\hline 40 & 106 & $2345: 3$ & 5134 & 3 \\
\hline 41 & 118 & $245: 34$ & 5186 & 1 \\
\hline 42 & 122 & $345: 23$ & 5246 & 4 \\
\hline 43 & 114 & $2345: 4$ & 5290 & 3 \\
\hline 44 & 105 & $234: 34$ & 5469 & 1 \\
\hline 45 & 127 & $345: 35$ & 5552 & 2 \\
\hline 46 & 126 & $345: 34$ & 5906 & 3 \\
\hline
\end{tabular}

${ }^{\mathrm{a}}$ : Values rounded off from Table 2 .

b: Most abundant ions of isotope clusters of $([\mathrm{M}-\mathrm{Cl}] /[\mathrm{M}]) \times 100 \%$.

c, d, e, f, g: Groups of isomers with separations of 5 sec or less.

h: "Ortho effect" used with the elution order can distinguish these nearest neighbors.

h: From groups c, d, e, f, g, these coeluters can be distinguished using the "ortho effect." 
TABle 4: Congeners and Some PCB Coeluting Isomers (1D Mode) with their Percent "Ortho Effects".

IUPAC Numbers of Coeluting Isomers ("Ortho Effects")

\begin{tabular}{lc}
\hline Tri & $28(5 \%)+33(5 \%)+21(6 \%)$ \\
Tri & $39(5 \%)+38(7 \%)$ \\
Tetra & $64(4 \%)+40(82 \%)^{*}$ \\
Tetra & $65(7 \%)+62(5 \%)+47(26 \%)^{*}$ \\
Tetra & $67(3 \%)+58(4 \%)$ \\
Hexa & $163(4 \%)+129(56 \%)^{*}$ \\
Hepta & $182(32 \%)+175(44 \%)$ \\
Octa & $201(4 \%)+204(2 \%)$ \\
\hline
\end{tabular}

* This isomer can be distinguished from other coeluting isomers by the "ortho effect." The penta isomers were given previously in the text and figures.

However, as illustrated in Figure 2, PCB congeners numbers 4 and 10 (2,2' - and 2,6-dichlorobiphenyl, resp.) were ultimately distinguished by taking advantage of their "orthoeffect" (38). The 2,2'-dichlorobiphenyl had a higher abundance of the $m / z 187(\mathrm{M}-\mathrm{Cl})^{+}$ion relative to the molecular ion at $m / z 222$. This fragment was not nearly as significant in 2,6-dichlorobiphenyl, where the two ortho chlorines were on the same ring. Note, there was partial resolution in this case. For complete coelution, if the magnitude of the "ortho effect" were known for each isomer, and were sufficiently different, it would be possible to estimate the relative concentrations of each isomer present. For example, if equal concentrations of each isomer were present, the observed "ortho effect" would be expected to be half way between the two values.

It was observed that the PCB congener retention times in the 1D chromatographic mode were different by a few seconds from those of the $2 \mathrm{D}$ retention times due to the modulation effect. Based on our analytical conditions, as stated previously, the isomeric peak separations, achieved using the $2 \mathrm{D}$ chromatographic mode, occurred within approximately $2-3 \mathrm{sec}$ on the $2 \mathrm{D}$ plane. When the instrument was optimized (setting the trapping time to 1.2 secs) for pentachlorobiphenyl isomer separations (Figure 1) in a different $2 \mathrm{D}$ run, the isomeric peak separations occurred at about 1$2 \mathrm{sec}$ on the 2D plane. The significance of this adjustment was that most of the potential interferences would be separated twice from the target analytes: once, mostly volatile interferences, in the first $1000 \mathrm{sec}$ of the $40 \mathrm{~m}$ column of the 1D plane, and second, interfering hydrocarbons, on the first $1 \mathrm{sec}$ of the $1 \mathrm{~m}$ column of the 2D plane (Figure 1).

The early elution of these potential interferences would leave the analytes of interest in a purer form, resulting in more accurate full-scan library identification. This tool may prove very useful for the identification of individual $\mathrm{PCB}$ congeners in extracts of complex mixtures such as sewage treatment plant sludge or river sediments.

Table 4 shows 18 PCBs that were not distinguishable using the $1 \mathrm{D}$ chromatographic mode and three of these 18 PCB congeners were additionally distinguished from their isomers, using the "ortho effect." This further raised the numbers of distinguishable PCB congeners by three. As indicated earlier in this paper (see Figure 2), PCB isomers 4 and 10 (2,2' - and 2,6-dichlorobiphenyl, resp.) were distinguished by their "ortho-effect," and this also increased the total number of distinguishable congeners by one. In spite of the use of the GC $\times \mathrm{GC}$-TOFMS, not all of the congeners were distinguishable. The $2 \mathrm{D}$ chromatographic conditions permitted the separation of most of the pentachlorobiphenyls, but this was found to be at the expense of resolving the tetrachlorobiphenyls (e.g., IUPAC \#62 and \#65). It was necessary to optimize the system for those separations of interest. Based on these experiments, GC $\times$ GC-TOFMS provided more complete separation of isomeric $\mathrm{PCBs}$ that were unresolved after the 1D mode was used. Even though not all 209 PCB congeners [33] were chromatographically resolved, the use of the $2 \mathrm{D}$ chromatographic mode provided superior data for assessing the risk posed by a complex mixture of PCBs. We believe that complete separation of all 209 PCB congeners may eventually be achieved by two consecutive GC runs and with the advent of enhanced columns and different chromatographic conditions, optimized to resolve the most difficult to separate PCB isomers or congeners.

More chromatographic work and superior deconvolution software may be needed to take full advantage of this promising technology for isomeric compound separations. The authors used the default data resample feature of the software to resample large data files to reduce the file size and better define chromatographic peaks, which resulted in enhanced peak separations.

\section{Conclusion}

In light of recent scientific findings that noncoplanar PCB congeners were also culpable in human health deterioration, as well as planar PCBs, we felt it was important to develop a method that would potentially separate all possible PCB congeners, which would be ready for use on demand. It would be highly unlikely for all congeners to be simultaneously present in any given environmental sample, for example, heavily chlorinated biphenyls with all chlorines on one ring and none on the other ring would be inconsistent with the known electrophilic method of preparation of the Aroclors. However, these or other non-Aroclor PCB congeners, could result from different processes. Because we have examined all PCB congeners, this study has included all those that were found to be ubiquitous, toxic, or potentially toxic. This study considered all congeners that would result from all possible routes: intentional, or incidental, chlorinated biphenyl syntheses, for example, coupling reactions, and as byproducts, and environmental alteration products by selective vaporization or absorption, and reductive dechlorination during metabolism, photochemical reaction, combustion, and chemical remediation [44-47]. Rather than relying on the common method of total or combined PCB quantification, or the electron capture detector (for high sensitivity), this study employed technology that provided excellent separation of 196 PCB congeners that were either present in or absent from Aroclor formulations. Indeed, the 

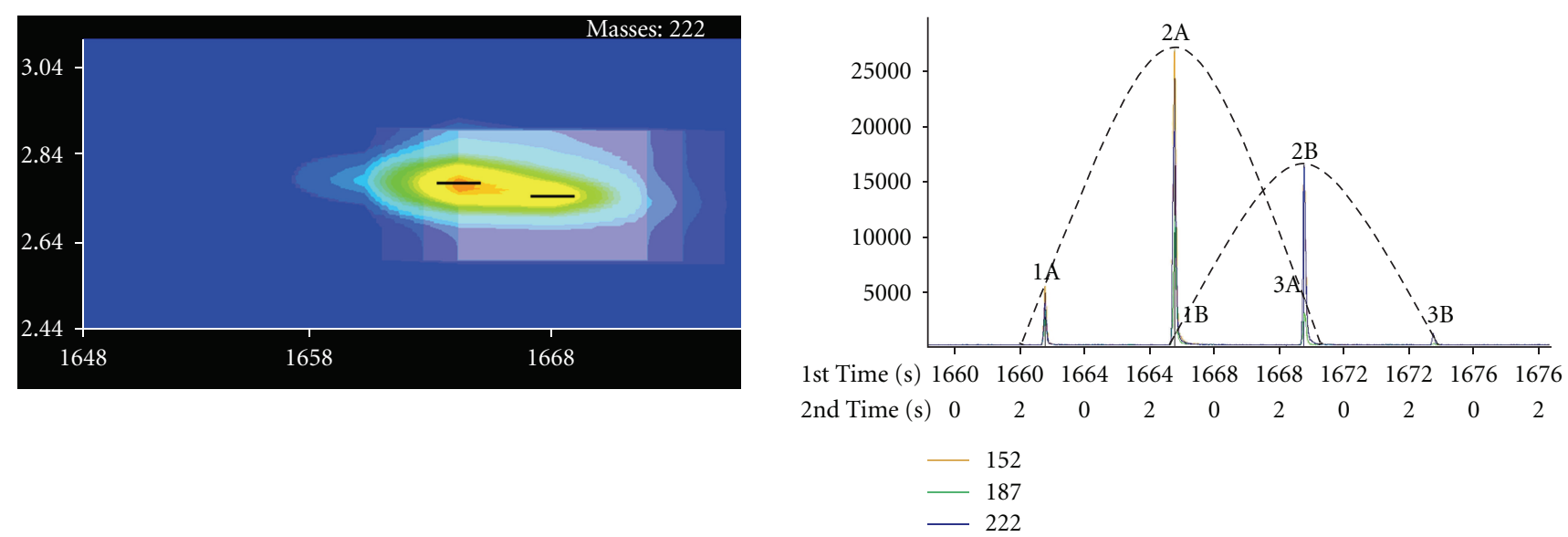

(a)
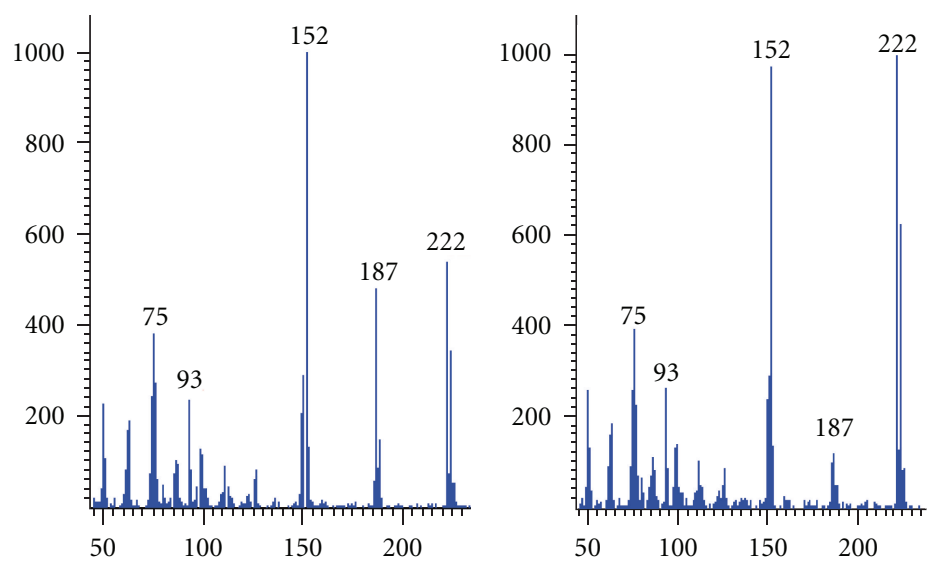

(c)

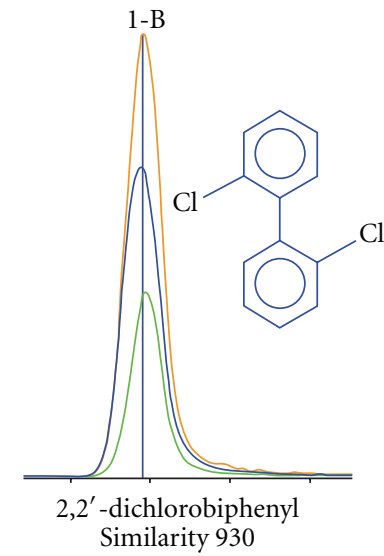

$\mathrm{Cl}$

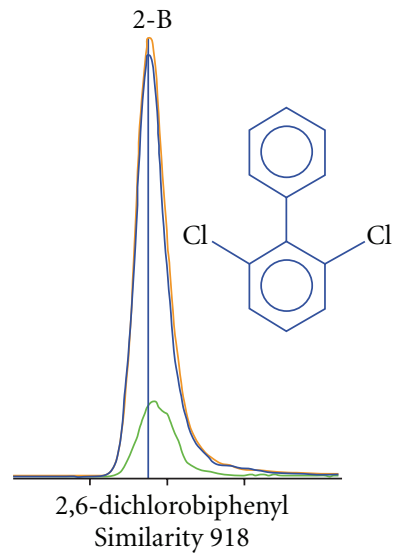

(d)

FIGURE 2: Use of chromatographic and mass spectrometric data in a case of isomer coelution. (a) Contour plot from above looking down on a $2 \mathrm{D}$ run of the coeluting 2,2' - and 2,6-dichlorobiphenyls. (b) Chromatogram of the overlapping isomers viewed from the side and showing three slices each, taken from the 2D mode of separation. (c) The mass spectra obtained from pure standards of the individual isomers, each with two ortho chlorines, but one having these chlorines on separate rings $\left(2,2^{\prime}-\right.$ ) and showing the mass spectrometric "ortho effect." (d) Mass chromatograms of the isomer mixture showing $m / z 222$ (blue), $m / z 187$ (green), and $m / z 152$ (red) and the partial resolution and assignment of the isomers based on these relative intensities.

need to overcome the challenges of individual PCB congener separation, in not more than two chromatographic runs, remained an approachable, but not yet achieved goal. The quest for a congener-specific analysis of all PCB congeners in complex environmental mixtures, without sacrificing separation quality, is on-going and this paper presents a step towards this goal.

\section{Acknowledgments}

The authors acknowledge Dr. Christian G. Daughton for initiating this research and Dr. Mark Libardoni for helping with the initial operation of the instrument. Thanks also to Mrs. Nellie Dujua for assisting with the PCB congener standards preparation and manual measurement of the "ortho effect."
Leco is gratefully acknowledged here for collaborating with the U.S. EPA and providing the instrument for this research. The US Environmental Protection Agency (EPA), through its Office of Research and Development (ORD), funded and performed the research described. This paper has been subjected to the EPA's peer and administrative review and has been approved for publication. Mention of trade names or commercial products in this paper does not constitute endorsement or recommendation by the EPA.

\section{References}

[1] J. W. Cochran and G. M. Frame, "Recent developments in the high-resolution gas chromatography of polychlorinated biphenyls," Journal of Chromatography A, vol. 843, no. 1-2, pp. 323-368, 1999. 
[2] B. R. Larson, "HRGC separation of PCB congeners," Journal of High Resolution Chromatography, vol. 18, pp. 141-151, 1995.

[3] G. M. Frame, J. W. Cochran, and S. S. Bøwadt, "Complete PCB Congener Distributions for 17 Aroclor Mixtures Determined by 3 HRGC Systems Optimized for Comprehensive, Quantitative, Congener-Specific Analysis," HRC Journal of High Resolution Chromatography, vol. 19, no. 12, pp. 657-668, 1996.

[4] U.S. EPA, "Method 1668, revision a: chlorinated biphenyl congeners in water, soil, sediment, and tissue by HRGC/ HRMS," EPA no. EPA-821-r-00-002, 1999, http://www.epa .gov/region3/1668a.pdf.

[5] T. Kenet, R. C. Froemke, C. E. Schreiner, I. N. Pessah, and M. M. Merzenich, "Perinatal exposure to a noncoplanar polychlorinated biphenyl alters tonotopy, receptive fields, and plasticity in rat primary auditory cortex," Proceedings of the National Academy of Sciences of the United States of America, vol. 104, no. 18, pp. 7646-7651, 2007.

[6] L. G. Hansen, "Stepping backward to improve assessment of PCB congener toxicities," Environmental Health Perspectives, vol. 106, no. 1, pp. 171-189, 1998.

[7] V. A. McFarland and J. U. Clarke, "Environmental occurrence, abundance, and potential toxicity of polychlorinated biphenyl congeners: considerations for a congener-specific analysis," Environmental Health Perspectives, vol. 81, pp. 225-239, 1989.

[8] E. Hoh, K. Mastovska, and S. J. Lehotay, "Optimization of separation and detection conditions for comprehensive twodimensional gas chromatography-time-of-flight mass spectrometry analysis of polychlorinated dibenzo-p-dioxins and dibenzofurans," Journal of Chromatography A, vol. 1145, no. 1-2, pp. 210-221, 2007.

[9] D. R. Rushneck, A. Beliveau, B. Fowler et al., "Concentrations of dioxin-like PCB congeners in unweathered Aroclors by HRGC/HRMS using EPA Method 1668A," Chemosphere, vol. 54, no. 1, pp. 79-87, 2004.

[10] J. D. Berset and R. Holzer, "Determination of coplanar and ortho substituted PCBs in some sewage sludges of Switzerland using HRGC/ECD and HRGC/MSD," Chemosphere, vol. 32, no. 12 , pp. 2317-2333, 1996.

[11] L. F. de Alencastro, D. Grandjean, and J. Tarradellas, "Application of multidimensional (heart-cut) gas chromatography to the analysis of complex mixtures of organic pollutants in environmental samples," Chimia, vol. 57, no. 9, pp. 499-504, 2003.

[12] G. M. Frame, "A collaborative study of 209 PCB congeners and 6 Aroclors on 20 different HRGC columns: 1 . Retention and coelution database," Fresenius' Journal of Analytical Chemistry, vol. 357, no. 6, pp. 701-713, 1997.

[13] E. Reiner, A. Boden, K. MacPherson, T. Kolic, F. Dorman, and J. Cochran, "Chromatographic enhancement techniques for the analysis of persistent organic pollutants in environmental samples," Organohalogen Compound, vol. 66, pp. 838-845, 2004.

[14] J. W. Cochran, "Fast gas chromatography-time-of-flight mass spectrometry of polychlorinated biphenyls and other environmental contaminants," Journal of Chromatographic Science, vol. 40, no. 5, pp. 254-268, 2002.

[15] U.S. EPA, "Polychlorinated biphenyls (PCBs) by gas chromatography," 2000, pp.1-57, http://www.horizontechinc.com/ htmpages/EPAMethods/Method_8082a.pdf.

[16] U.S. EPA, "Polychlorinated biphenyls (PCBs) by gas chromatography," 2007, pp.1-56, http://www.epa.gov/osw/hazard/ testmethods/sw846/pdfs/8082a.pdf.
[17] D. L. Swackhamer and D. E. Armstrong, "Distribution and characterization of PCBs in Lake Michigan water," Journal of Great Lakes Research, vol. 13, no. 1, pp. 24-36, 1987.

[18] S. S. Bøwadt and B. Larsen, "Rapid screening of chlorobiphenyl congeners by GC-ECD on a carborane-polydimethylsiloxane copolymer," Journal of High Resolution Chromatography, vol. 15, pp. 350-351, 1992.

[19] S. H. Safe, "Polychlorinated biphenyls (PCBs): environmental impact, biochemical and toxic responses, and implications for risk assessment," Critical Reviews in Toxicology, vol. 24, no. 2, pp. 87-149, 1994.

[20] C. Vendeuvre, R. Ruiz-Guerrero, F. Bertoncini, L. Duval, and D. Thiébaut, "Comprehensive two-dimensional gas chromatography for detailed characterisation of petroleum products," Oil and Gas Science and Technology, vol. 62, no. 1, pp. 43-55, 2007.

[21] J. Dallüge, M. van Rijn, J. Beens, R. J. J. Vreuls, and U. A. T. Brinkman, "Comprehensive two-dimensional gas chromatography with time-of-flight mass spectrometric detection applied to the determination of pesticides in food extracts," Journal of Chromatography A, vol. 965, no. 1-2, pp. 207-217, 2002.

[22] J. F. Focant, G. Eppe, M. L. Scippo et al., "Comprehensive twodimensional gas chromatography with isotope dilution timeof-flight mass spectrometry for the measurement of dioxins and polychlorinated biphenyls in foodstuffs: comparison with other methods," Journal of Chromatography A, vol. 1086, no. 1-2, pp. 45-60, 2005.

[23] S. Morales-Muñoz, R. J. J. Vreuls, and M. D. Luque De Castro, "Dynamic ultrasound-assisted extraction of environmental pollutants from marine sediments for comprehensive twodimensional gas chromatography with time-of-flight mass spectrometric detection," Journal of Chromatography A, vol. 1086, no. 1-2, pp. 122-127, 2005.

[24] P. Marriott and R. Shellie, "Principles and applications of comprehensive two-dimensional gas chromatography," TrACTrends in Analytical Chemistry, vol. 21, no. 9-10, pp. 573-583, 2002.

[25] R. Shellie, P. Marriott, and P. Morrison, "Concepts and preliminary observations on the triple-dimensional analysis of complex volatile samples by using GC $\times$ GC-TOFMS," Analytical Chemistry, vol. 73, no. 6, pp. 1336-1344, 2001.

[26] J. Beens and U. A. T. Brinkman, "Comprehensive twodimensional gas chromatography-a powerful and versatile technique," Analyst, vol. 130, no. 2, pp. 123-127, 2005.

[27] J. B. Phillips and J. Xu, "Comprehensive multi-dimensional gas chromatography," Journal of Chromatography A, vol. 703, no. 1-2, pp. 327-334, 1995.

[28] J. Beens, R. Tijssen, and J. Blomberg, "Prediction of comprehensive two-dimensional gas chromatographic separations. A theoretical and practical exercise," Journal of Chromatography A, vol. 822, no. 2, pp. 233-251, 1998.

[29] M. Harju, C. Danielsson, and P. Haglund, "Comprehensive two-dimensional gas chromatography of the 209 polychlorinated biphenyls," Journal of Chromatography A, vol. 1019, no. 1-2, pp. 111-126, 2003.

[30] G. Semard, A. Bruchet, P. Cardinaël, and J. P. Bouillon, "Use of comprehensive two-dimensional gas chromatography for the broad screening of hazardous contaminants in urban wastewaters," Water Science and Technology, vol. 57, no. 12, pp. 1983-1989, 2008.

[31] W. Bertsch, "Two-dimensional gas chromatography. Concepts, instrumentation, and applications: part 2: comprehensive two-dimensional gas chromatography," HRC Journal of 
High Resolution Chromatography, vol. 23, no. 3, pp. 167-181, 2000.

[32] P. Korytár, P. E. G. Leonards, J. De Boer, and U. A. T. Brinkman, "High-resolution separation of polychlorinated biphenyls by comprehensive two-dimensional gas chromatography," Journal of Chromatography A, vol. 958, no. 1-2, pp. 203-218, 2002.

[33] J. F. Focant Jr., A. Sjödin, and D. G. Patterson, "Improved separation of the 209 polychlorinated biphenyl congeners using comprehensive two-dimensional gas chromatographytime-of-flight mass spectrometry," Journal of Chromatography A, vol. 1040, no. 2, pp. 227-238, 2004.

[34] NIST/EPA/NIH, "Mass spectral library. Mass Spectral Library with Search Program (Data Version: NIST 05, Software Version 2.0d) purchased through Agilent technologies," Wilmington, Del, USA.

[35] E. O. Oswald, L. Levy, B. J. Corbett, and M. P. Walker, "Differentiation and characterization of isomeric polychlorinated biphenyls by gas liquid chromatography coupled with electron impact and chemical ionization mass spectrometry," Journal of Chromatography, vol. 93, no. 1, pp. 63-90, 1974.

[36] L. A. Levy and E. O. Oswald, "The effect of ortho substitution on the mass spectral fragmentation of polychlorinated biphenyls," Biomedical Mass Spectrometry, vol. 3, no. 2, pp. 8890, 1976.

[37] G. W. Sovocool and N. K. Wilson, "Differentiation of brominated biphenyls by carbon-13 nuclear magnetic resonance and gas chromatography/mass spectrometry," Journal of Organic Chemistry, vol. 47, no. 21, pp. 4032-4037, 1982.

[38] G. W. Sovocool, R. K. Mitchum, and J. R. Donnelly, "Use of the 'ortho effect' for chlorinated biphenyl and brominated biphenyl isomer identification," Biomedical \& environmental mass spectrometry, vol. 14, no. 10, pp. 579-582, 1987.

[39] M. D. Mullin, C. M. Pochini, S. McCrindle, M. Romkes, S. H. Safe, and L. M. Safe, "High-resolution PCB analysis: synthesis and chromatographic properties of all 209 PCB congeners," Environmental Science and Technology, vol. 18, no. 6, pp. 468476, 1984.

[40] J. Lulek, M. Opielewicz, K. Szyrwińska, and B. Milanowski, "Application of a retention database to the identification of individual polychlorinated biphenyl congeners in Aroclors mixture using selected polychlorinated biphenyls as a reference series," Analytica Chimica Acta, vol. 540, no. 1, pp. 25-31, 2005.

[41] Restek, "PCB congener retention data:Application note \#59120. PCB Congener Retention Data: application Note \#59120," http://www.restekcorp.com.

[42] K. Ballschmiter and M. Zell, "Analysis of polychlorinated biphenyls (PCB) by glass capillary gas chromatographycomposition of technical aroclor- and Clophen-PCB mixtures," Fresenius' Zeitschrift für Analytische Chemie, vol. 302, no. 1, pp. 20-31, 1980.

[43] S. A. Mills, D. I. Thal, and J. Barney, "A summary of the 209 PCB congener nomenclature," Chemosphere, vol. 68, no. 9, pp. 1603-1612, 2007.

[44] V. S. Magar, G. W. Johnson, R. C. Brenner et al., "Longterm recovery of $\mathrm{PCB}$-contaminated sediments at the Lake Hartwell superfund site: PCB dechlorination. 1. End-member characterization," Environmental Science and Technology, vol. 39, no. 10, pp. 3538-3547, 2005.

[45] J. W. Farrington, M. C. Loehr, E. L. Anderson, F. Bohle, Y. Cohen, and K. J. Farley, "Executive summary," in A risk Management Strategy for PCB-Contaminated Sediments, J. W. Farrington, Ed., pp. 1-16, 2001.
[46] M. Lores, M. Llompart, R. González-García, C. GonzálezBarreiro, and R. Cela, "On-fibre photodegradation studies of polychlorinated biphenyls using SPME-GC-MS-MS: a new approach," Chemosphere, vol. 47, no. 6, pp. 607-615, 2002.

[47] C. S. Wong, A. W. Garrison, and W. T. Foreman, "Enantiomeric composition of chiral polychlorinated biphenyl atropisomers in aquatic bed sediment," Environmental Science and Technology, vol. 35, no. 1, pp. 33-39, 2001. 


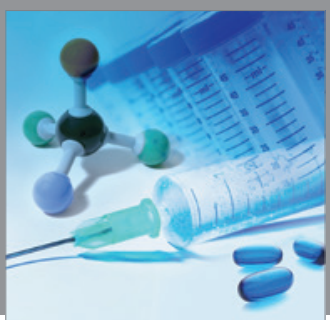

International Journal of

Medicinal Chemistry

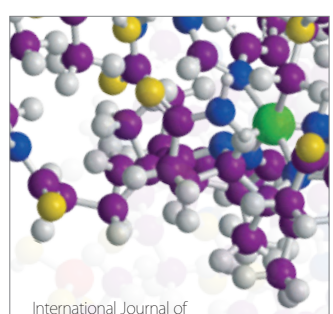

Carbohydrate Chemistry

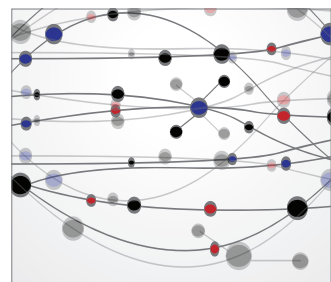

The Scientific World Journal
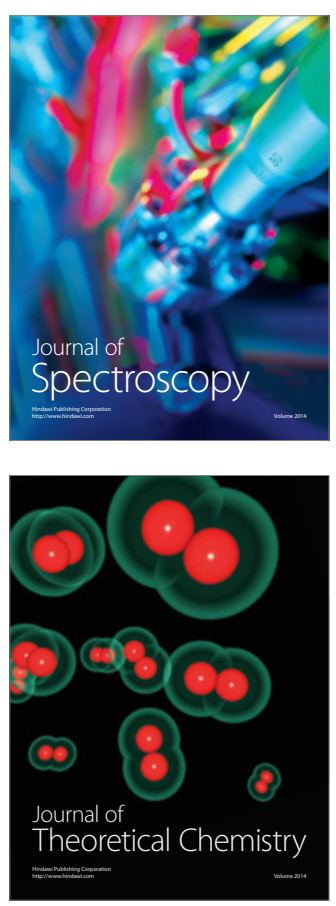
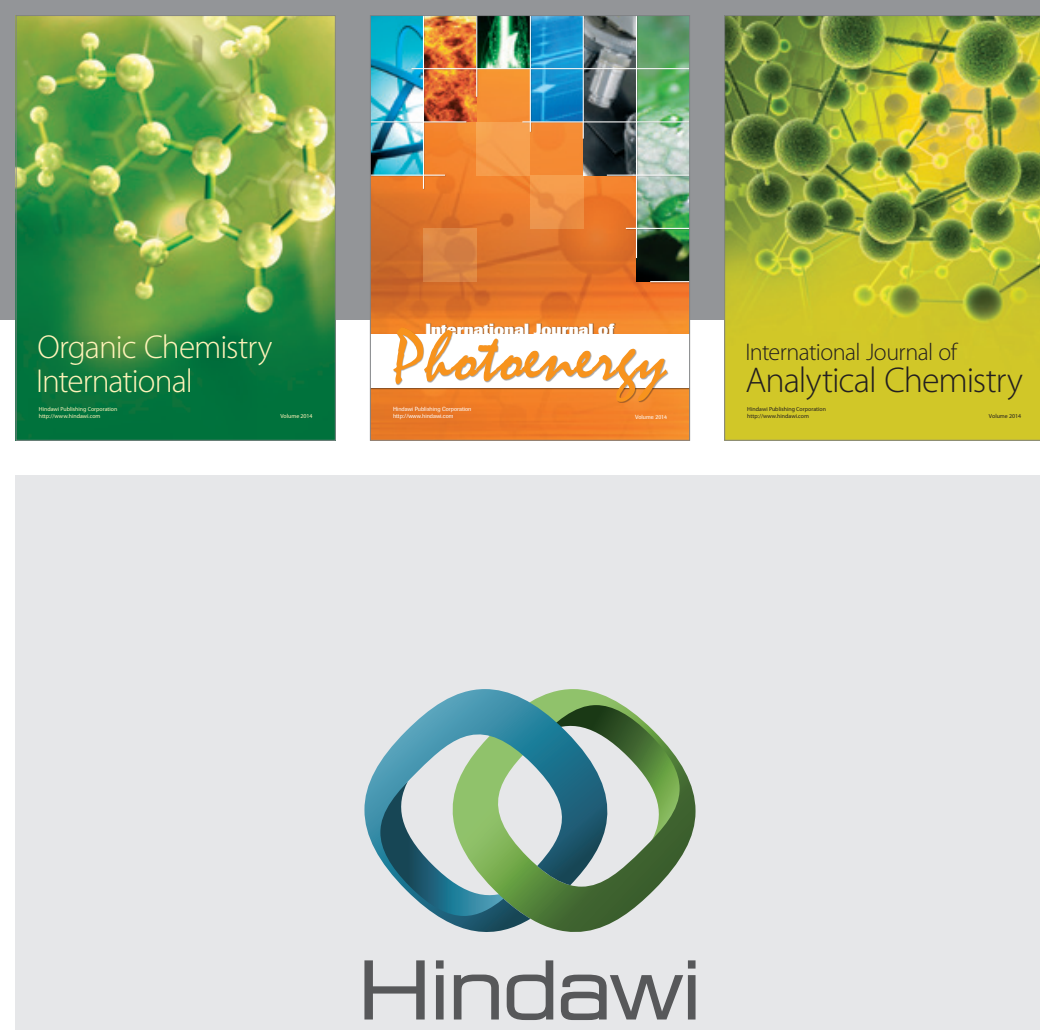

Submit your manuscripts at

http://www.hindawi.com
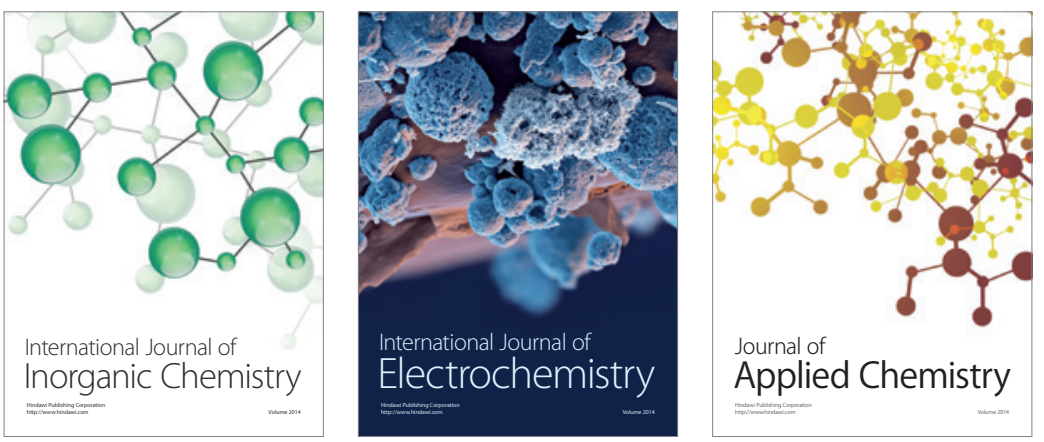

Journal of

Applied Chemistry
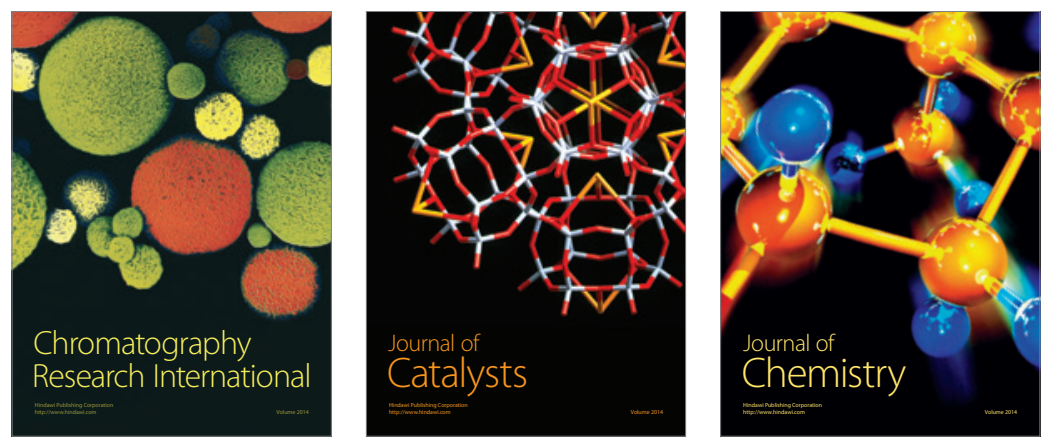
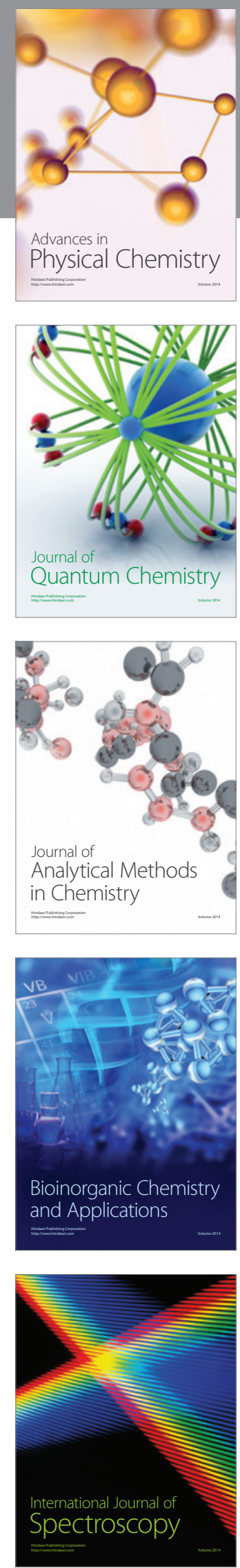